\title{
AN ESTIMATE FOR THE ENUMERATIVE FUNCTIONS OF CERTAIN SETS OF INTEGERS ${ }^{1}$
}

\section{R. L. ROBINSON}

1. Introduction. It is the purpose of this note to generalize several related results in the theory of numbers due to E. Cohen. In particular, Theorem 1 of this paper contains as special cases Theorems 2.1, 3.1 , and 4.1 of [2]. A corollary of Theorem 1 gives an estimate for the enumerative function $N(x) \equiv \sum 1(1 \leqq r \leqq x, r \in N)$ of certain integer sequences $N$.

Let $k$ denote a fixed integer $\geqq 2$, and denote by $L_{k}$ the set of all integers $r>0$ each prime divisor of which has multiplicity $\geqq k$. The integer 1 is taken to be in $L_{k}$ for every $k$. For integral $r>0, L_{k}(r)$ is defined to be the largest divisor of $r$ in $L_{k}$. In $\$ 3$ Theorem 1 is applied to a discussion of the sequence of integers $r$ for which $L_{k}(r)$ has specified properties. If $r=p_{1}^{e_{1}} \cdots p_{m}^{e_{m}}$ is the canonical factorization of $r$ and if the function $\Delta$ is defined by

$$
\Delta(r)=\left(e_{1}-1\right)+\left(e_{2}-1\right)+\cdots+\left(e_{m}-1\right) \quad(\Delta(1)=0),
$$

then a consequence of this discussion in the case $k=2$ is the formula

$$
d(n)=\frac{6}{\pi^{2}} \sum_{\Delta(r)=n} \frac{l_{2}(r)}{\Psi(r)}
$$

for the density $d(n)$ of the sequence $D_{n}$ consisting of all $r>0$ for which $\Delta(r)=n$. In this formula $l_{2}$ is the characteristic function of $L_{2}$, i.e., $l_{2}(r)=1$ or 0 according as $r \in L_{2}$ or $r \notin L_{2}, \Psi(r)$ is Dedekind's $\Psi$-function defined by (6), and the summation is over all $r$ for which $\Delta(r)=n$. The sequence $D_{n}$ was originally studied by A. Rényi [5] who showed that

$$
\sum_{n=0}^{\infty} d(n) z^{n}=\frac{6}{\pi^{2}} \prod_{p}\left(1+\frac{z}{(p+1)(p-z)}\right), \quad|z|<2 .
$$

In $\S 3$ a generalization of this formula will be indicated.

For a discussion of the sequence $D_{n}$ and a derivation of (3) from a statistical point of view see Kac's book [4]. It is interesting to note that, contrary to Kac's remark, the rather elegant explicit formula (2) for the density $d(n)$ can be obtained.

Received by the editors December 17, 1964.

1 This research was supported in part by the National Science Foundation Grant GP-1632. 
For further applications of Theorem 1 the reader is referred to [6].

2. Main results. For this section the following notation will be adopted: $Z$ will denote the set of all positive integers; $A$ and $B$ are subsets of $Z ; S, T$, and $U$, sets of complex numbers; $f$ and $g$, functions from $Z \times Z$ to the complex numbers; and $\rho$, a function from $Z$ to the complex numbers. The $O$-estimates of this section and the next are intended for all $x$ sufficiently large.

Lemma 1. Let sets $B, T, U$ and functions $f$ and $g$ be given. Denote by $\beta$ the characteristic function of $B$. Assume that there exist non-negative functions $K$ and $M$ and numbers $\delta$ and $\epsilon$ with $\epsilon \geqq 0$ and $1>\delta \geqq 0$ such that

$$
\sum_{r \leq x ; f(r, n) \in T ; g(r, n) \in U} \beta(r)=K(n) x+O\left(M(n) x^{\delta} \log ^{\boldsymbol{e}} x\right) .
$$

Then for any $\alpha \geqq 0$

$\sum_{r \leqslant x ; f(r, n) \in T ; g(r, n) \in U} r^{\alpha} \beta(r)=K(n) \frac{x^{\alpha+1}}{\alpha+1}+O\left((K(n)+M(n)) x^{\alpha+\delta} \log ^{\epsilon} x\right)$.

The proof is straightforward; details are given in [6].

A special case of Lemma 1 merits mention.

Corollary. $(f(r, n)=g(r, n)=(r, n) ; T=U=\{1\}$. $)$ If

$$
\sum_{r \leq x ;(r, n)=1} \beta(r)=K(n) x+O\left(M(n) x^{\delta} \log ^{e} x\right)
$$

with $0 \leqq \delta<1,0 \leqq \epsilon$, then for any $\alpha \geqq 0$

$$
\sum_{r \leq x ;(r, n)=1} r^{\alpha} \beta(r)=K(n) \frac{x^{\alpha+1}}{\alpha+1}+O\left((K(n)+M(n)) x^{\alpha+\delta} \log ^{e} x\right) .
$$

The principal result of this paper is the following theorem whose proof on the basis of Lemma 1 is completely analogous to the proof of Theorem 2.1 of [2]. (See also [6, §3].)

Theorem 1. Let $B, T, U, f$, and $g$ be specified so that the hypotheses of Lemma 1 are satisfied. Suppose also that there is a set $A$ such that every positive integer $r$ is uniquely factorable in the form

$$
r=a(r) b(r), \quad a(r) \in A, \quad b(r) \in B, \quad g(a(r), b(r)) \in U .
$$

Then for any $\alpha \geqq 0$ and any choice of $\rho$ and $S$ such that $\rho(r) \in S$ for at least oner 


$$
\begin{aligned}
C_{\rho \alpha}(x, S) & \equiv \sum_{r \leqslant x ; f(a(r), b(r)) \in T ; \rho(a(r)) \in S}(b(r))^{\alpha} \\
& =\frac{x^{\alpha+1}}{\alpha+1} \sum_{r \leq x ; r \in A ; \rho(r) \in S} \frac{K(r)}{r^{\alpha+1}}+O\left(R_{\rho \alpha}(x, S) x^{\alpha+\delta} \log ^{\epsilon} x\right)
\end{aligned}
$$

where

$$
R_{\rho \alpha}(x, S)=\sum_{r \leqq x ; r \in A ; \rho(r) \in S} \frac{K(r)+M(r)}{r^{\alpha+\delta}} .
$$

CoROLlary 1.1. Under the hypotheses of Theorem 1 the number of integers $\leqq x$ such that $f(a(r), b(r)) \in T$ and $\rho(a(r)) \in S$ is

$$
C_{\rho 0}(x, S)=x \sum_{r \unlhd x ; r \in A ; \rho(r) \in S} \frac{K(r)}{r}+O\left(R_{\rho 0}(x, S) x^{\delta} \log ^{e} x\right) .
$$

Moreover, if $\sum K(r) / r(r \in A, \rho(r) \in S)$ converges and $R_{\rho 0}(x, S)=O\left(x^{t}\right)$ for some $t<\delta$, then the density of the set of all $r$ such that $f(a(r), b(r)) \in T$ and $\rho(a(r)) \in S$ is given by $\sum K(r) / r(r \in A, \rho(r) \in S)$.

3. An application. Corollary 1.1 appears to be limited in scope. Nevertheless, when it is applicable it provides a convenient tool for studying the sequence of integers $r$ for which $a(r)$ has a given property. One such application will be indicated here.

For integral $k \geqq 2$ let $Q_{k}$ denote the set of all $r>0$ such that each prime factor of $r$ has multiplicity $<k$. The integers in $Q_{k}$ will be called $k$-free integers. The integer 1 is taken to be $k$-free for every $k$. Clearly, every positive integer $r$ is uniquely factorable in the form

$$
r=L_{k}(r) Q_{k}(r), \quad L_{k}(r) \in L_{k}, \quad Q_{k}(r) \in Q_{k}, \quad\left(L_{k}(r), Q_{k}(r)\right)=1
$$

where $L_{k}$ is defined in the introduction. Furthermore, if $q_{k}$ is the characteristic function of $Q_{k}$ and

$$
\Psi_{k}(r) \equiv r \prod_{p \mid r}\left(1+p^{-1}+p^{-2}+\cdots+p^{-(k-1)}\right),
$$

then by obvious modifications of the proof of Lemma 5.2 of [1] one obtains the following lemma, which shows that the hypotheses of Theorem 1 are satisfied if $A=L_{k}, B=Q_{k}, T=Z, U=\{1\}, f(r, n)$ $=g(r, n)=(r, n)$.

Lemma 2. If $\theta(n)$ denotes the number of divisors of $n$ which belong to $Q_{2}$ and $q_{k}$ is the characteristic function of $Q_{k}$, then 


$$
\sum_{r \leqq x ;(r, n)=1} q_{k}(r)=\frac{n}{\zeta(k) \Psi_{k}(n)} x+O\left(\theta(n) x^{1 / k}\right),
$$

where $\Psi_{k}$ is given by (5) and $\zeta$ is the Riemann zeta-function.

Furthermore, if $l_{k}$ is the characteristic function of $L_{k}$, then using the estimates $\sum_{r \leqq x} l_{k}(r)=c(k) x^{1 / k}+O\left(x^{1 /(k+1)}\right) \quad[3], \theta(r)=O\left(r^{t}\right)$ for arbitrary $t>0[1, \S 3]$, and $r / \Psi_{k}(r)=O(1)$, one can show by partial summation that $\sum_{r=1}^{\infty} l_{k}(r) / \Psi_{k}(r)$ converges and

$$
R_{\rho \alpha} \equiv \sum_{r \leq x ; \rho(r) \in S} l_{k}(r)\left(\theta(r)+r / \zeta(k) \Psi_{k}(r)\right) r^{-(\alpha+1 / k)}= \begin{cases}O(1) & \text { if } \alpha>0, \\ O\left(x^{\epsilon}\right) & \text { if } \alpha=0,\end{cases}
$$

for any function $\rho$ and set $S$ and every $\epsilon>0$. Therefore, Theorem 1 implies the following result.

THEOREM 2. If $Q_{k}(r)$ is defined by (4), then for any function $\rho$, any $\alpha \geqq 0$, and any $S$ for which $\rho(r) \in S$ for at least one $r$

$$
\begin{aligned}
\sum_{r \leqq x ; \rho\left(L_{\boldsymbol{x}}(r)\right) \in S}\left(Q_{k}(\boldsymbol{r})\right)^{\alpha}= & \frac{x^{\alpha+1}}{\zeta(k)(\alpha+1)} \sum_{\rho(r) \in S} \frac{l_{k}(\boldsymbol{r})}{r^{\alpha} \Psi_{k}(r)} \\
& + \begin{cases}O\left(x^{\alpha+1 / k}\right) & \text { if } \alpha>0, \\
O\left(x^{++1 / k}\right) & \text { if } \alpha=0,\end{cases}
\end{aligned}
$$

for every $\epsilon>0$.

Take $\rho(r)=r, S=Z, \alpha=1$. Since $Q_{k}(r)$ is the largest unitary $k$-free divisor of $r$, i.e., the largest $k$-free divisor of $r$ which is relatively prime to its conjugate divisor, we obtain

Corollary 2.1 .

$$
\frac{1}{x} \sum_{r \leqq x} Q_{k}(r)=\frac{x}{2 \zeta(k)} \sum_{r=1}^{\infty} \frac{l_{k}(r)}{r \Psi_{k}(r)}+O\left(x^{1 / k}\right) .
$$

In other words, the average order of the largest unitary $k$-free divisor of an integer is $(x / 2 \zeta(k)) \sum_{r=1}^{\infty} l_{k}(r) / r \Psi_{k}(r)$.

Now let $\rho(r)=r, S=Z, \alpha=0$. Then Theorem 2 implies the following corollary.

Corollary 2.2. If $l_{k}$ is the characteristic function of $L_{k}$ and $\Psi_{k}$ is defined by (5), then

$$
\sum_{r=1}^{\infty} \frac{l_{k}(r)}{\Psi_{k}(r)}=\zeta(k)
$$


In Theorem 2 put $S=\{n\}, n$ integral, $n \geqq 0$ and let $\rho=\Delta$, where $\Delta$ is defined in the introduction. Then with $\alpha=0$ we get

Corollary 2.3. For every $\epsilon>0$,

$$
\sum_{r \leq x ; \Delta\left(L_{k}(r)\right)=n} 1=\frac{x}{\zeta(k)} \sum_{\Delta(r)=n} \frac{l_{k}(r)}{\Psi_{k}(r)}+O\left(x^{\epsilon+1 / k}\right) .
$$

In particular, the density $d_{k}(n)$ of the set of all $r$ such that $\Delta\left(L_{k}(r)\right)=n$ is $(1 / \zeta(k)) \sum l_{k}(r) / \Psi_{k}(r)$ where the summation extends over all $r$ such that $\Delta(r)=n$.

Note, however, that $\Delta\left(L_{2}(r)\right)=\Delta(r)$. Hence, if

$$
\Psi(r) \equiv \Psi_{2}(r)=r \prod_{p \mid r}(1+1 / p)=r \sum_{d \mid r} \mu^{2}(d) / d
$$

where $\mu$ is Mobius' function, we obtain the result stated in the introduction.

CoRollary 2.4. For every $\epsilon>0$,

$$
\sum_{r \leq x ; \Delta(r)=n} 1=\frac{6 x}{\pi^{2}} \sum_{\Delta(r)=n} \frac{l_{2}(r)}{\Psi(r)}+O\left(x^{e+1 / 2}\right) .
$$

In particular, the density of the set of all $r$ such that $\Delta(r)=n$ is given by (2).

Finally, we note that a generalization of Rényi's formula (3) can be proved. With $d_{k}(n)$ defined as in Corollary 2.3,

$$
\sum_{n=0}^{\infty} d_{k}(n) z^{n}=\frac{1}{\zeta(k)} \prod_{p}\left(1+\frac{p-1}{p^{k}-1} \frac{z^{k-1}}{p-z}\right)
$$

for $|z|<2$. This result follows from a more general theorem concerning the factorization of power series into infinite products over primes [6, Theorems 1 and 3]. This topic will be treated in a later paper.

\section{REFERENCES}

1. E. Cohen, Arithmetical functions associated with the unitary divisors of an integer, Math. Z. 74 (1960), 66-80.

2. - Some asymptotic formulas in the theory of numbers, Trans. Amer. Math. Soc. 112 (1964), 214-227.

3. P. Erdös and G. Szekeres, Über die Anzahl der Abelschen Gruppen gegebener Ordnung und iiber ein verwandtes zahlentheoretisches Problem, Acta Szeged. 7 (1934), 95-102.

4. M. Kac, Statistical independence in probability, analysis, and number theory, Carus Math. Monographs, No. 12, Math. Assoc. of America, 1959. 
5. A. Rényi, On the density of certain sequences of integers, Acad. Serbe Sci. Publ. Inst. Math. 8 (1955), 157-162.

6. R. Robinson, An infinite product factorization for a class of generating functions defined by power series, Ph.D. dissertation, Univ. of Tennessee, Knoxville, Tenn., 1964.

UNIVERSITY OF TENNESSEE

\section{ANTIFLEXIBLE ALGEBRAS WHICH ARE NOT POWER-ASSOCIATIVE}

\section{J. RODABAUGH}

1. Introduction. An algebra $A$ will be called antiflexible if the elements of $A$ satisfy the identity

$$
(x, y, z)=(z, y, x) \text {. }
$$

Power-associative antiflexible algebras have been studied by the author [3] and Kosier [2].

As a matter of terminology, we shall define an algebra as a finite dimensional vector space on which a multiplication is defined in which both distributive laws are satisfied. If $A$ is an algebra over a field $F$ of characteristic not two, then $A$ has an attached algebra $A^{+}$ which is the same additive group as $A$ but the multiplication $x \cdot y$ of $A^{+}$is defined by

$$
x \cdot y=(x y+y x) / 2 .
$$

In any algebra $x^{n}$ is inductively defined by $x^{1}=x$ and $x^{k}=x^{k-1} x$. An algebra is power-associative if $x^{a} x^{b}=x^{a+b}$ for all $a, b$. The associator $(x, y, z)$ is defined as $(x y) z-x(y z)$ and the commutator $(x, y)$ is defined as $x y-y x$. For our purposes, $A$ is nodal if every member of $A$ can be written as $\alpha \cdot 1+z$ with $z$ nil in $A^{+}$.

Since we are not assuming power-associativity, we will define the radical as the maximal ideal of $A$ which is nil in $A^{+}$. An algebra is semisimple if its radical is zero. An algebra is simple if it is semisimple and if it contains no proper ideals. It should be clear that, in the case of power-associative antiflexible algebras, these definitions are equivalent to the ones in [2], [3].

\footnotetext{
Presented to the Society, August 31, 1964; received by the editors October 22,
} 1964. 